\title{
Dietary advanced glycation endproducts (AGEs) increase their concentration in plasma and tissues, result in inflammation and modulate gut microbial composition in mice; evidence for reversibility
}

Citation for published version (APA):

van Dongen, K. C. W., Linkens, A. M. A., Wetzels, S. M. W., Wouters, K., Vanmierlo, T., van de Waarenburg, M. P. H., Scheijen, J. L. J. M., de Vos, W. M., Belzer, C., \& Schalkwijk, C. G. (2021). Dietary advanced glycation endproducts (AGEs) increase their concentration in plasma and tissues, result in inflammation and modulate gut microbial composition in mice; evidence for reversibility. Food Research International, 147, [110547]. https://doi.org/10.1016/j.foodres.2021.110547

Document status and date:

Published: 01/09/2021

DOI:

10.1016/j.foodres.2021.110547

Document Version:

Publisher's PDF, also known as Version of record

Document license:

Taverne

Please check the document version of this publication:

- A submitted manuscript is the version of the article upon submission and before peer-review. There can be important differences between the submitted version and the official published version of record. People interested in the research are advised to contact the author for the final version of the publication, or visit the DOI to the publisher's website.

- The final author version and the galley proof are versions of the publication after peer review.

- The final published version features the final layout of the paper including the volume, issue and page numbers.

Link to publication

\footnotetext{
General rights rights.

- You may freely distribute the URL identifying the publication in the public portal. please follow below link for the End User Agreement:

www.umlib.nl/taverne-license

Take down policy

If you believe that this document breaches copyright please contact us at:

repository@maastrichtuniversity.nl

providing details and we will investigate your claim.
}

Copyright and moral rights for the publications made accessible in the public portal are retained by the authors and/or other copyright owners and it is a condition of accessing publications that users recognise and abide by the legal requirements associated with these

- Users may download and print one copy of any publication from the public portal for the purpose of private study or research.

- You may not further distribute the material or use it for any profit-making activity or commercial gain

If the publication is distributed under the terms of Article 25fa of the Dutch Copyright Act, indicated by the "Taverne" license above,

Download date: 26 Apr. 2023 


\title{
Dietary advanced glycation endproducts (AGEs) increase their concentration in plasma and tissues, result in inflammation and modulate gut microbial composition in mice; evidence for reversibility
}

\author{
Katja C.W. van Dongen ${ }^{\text {a,1 }}$, Armand M.A. Linkens ${ }^{\text {b,c, }}{ }^{2}$, Suzan M.W. Wetzels ${ }^{\text {c,d }}$, \\ Kristiaan Wouters $^{\text {b,c }}$, Tim Vanmierlo ${ }^{\text {e, }}$, Marjo P.H. van de Waarenburg ${ }^{\text {b,c }}$, Jean L.J. \\ M. Scheijen ${ }^{\text {, c }}$, Willem M. de Vos ${ }^{\mathrm{g}, \mathrm{h}}$, Clara Belzer ${ }^{\mathrm{g}, 2}$, Casper G. Schalkwijk ${ }^{\mathrm{b}, \mathrm{c}, *, 2}$ \\ ${ }^{a}$ Division of Toxicology, Wageningen University and Research, Stippeneng 4, 6708 WE Wageningen, the Netherlands \\ ${ }^{\mathrm{b}}$ Department of Internal Medicine, Maastricht University Medical Centre, Universiteitssingel 50, 6200MD Maastricht, the Netherlands \\ ${ }^{c}$ CARIM School for Cardiovascular Diseases, Maastricht University, Universiteitssingel 50, 6229ER Maastricht, the Netherlands \\ ${ }^{\mathrm{d}}$ Department of Pathology, Maastricht University Medical Centre, P. Debyelaan 25, 6229HX, the Netherlands \\ ${ }^{\mathrm{e}}$ Department of Psychiatry \& Neuropsychology, School for Mental Health and Neuroscience, Maastricht University, Dr Tanslaan 10, 6229ET Maastricht, the Netherlands \\ ${ }^{\mathrm{f}}$ Department of Neuroimmunology, Biomedical Research Institute, Hasselt University, Agoralaan gebouw D, BE3590 Diepenbeek, Belgium \\ ${ }^{\mathrm{g}}$ Laboratory of Microbiology, Wageningen University and Research, Stippeneng 4, 6708 WE Wageningen, the Netherlands \\ ${ }^{\mathrm{h}}$ Human Microbiome Research Program, Faculty of Medicine, University of Helsinki, Haartmaninkatu 3, Finland
}

\section{A R T I C L E I N F O}

\section{Keywords:}

Dietary advanced glycation endproducts Gut microbiota

16S rRNA sequencing

Ultra-performance liquid chromatography

tandem mass spectrometry

\begin{abstract}
A B S T R A C T
Scope: Dietary advanced glycation endproducts (AGEs) are associated with negative biological effects, possibly due to accumulation in plasma and tissues and through modulation of inflammation and gut microbiota. Whether these biological consequences are reversible by limiting dietary AGE intake is unknown.

Methods and results: Young healthy C57BL/6 mice were fed a standard chow $(\mathrm{n}=10)$ or a baked chow high AGEdiet $(\mathrm{n}=10)(\sim 1.8-6.9$ fold increased protein-bound Ne-(carboxymethyl)lysine (CML), NE-(1-carboxyethyl) lysine (CEL), and N8-(5-hydro-5-methyl-4-imidazolon-2-yl)-ornithine (MG-H1)) for 10 weeks or a switch diet with baked chow for 5 weeks followed by 5 weeks of standard chow $(n=10)$. We assessed accumulation of AGEs in plasma, kidney, and liver and measured inflammatory markers and gut microbial composition. After 10 weeks of baked chow, a substantial panel of AGEs were increased in plasma, liver, and kidney. These increases were normalized after the switch diet. The inflammatory z-score increased after the baked chow diet. Gut microbial composition differed significantly between groups, with enriched Dubosiella spp. dominating these alterations. Conclusion: A high AGE-diet led to an increase of AGEs in plasma, kidney, and liver and to more inflammation and modification of the gut microbiota. These effects were reversed or discontinued by a diet lower in AGEs.
\end{abstract}

Abbreviations: AGEs, Advanced glycation endproducts; ASV, amplicon sequence variants; CEL, Ne-(1-carboxyethyl)lysine; CML, Ne-(carboxymethyl)lysine; CR, c-

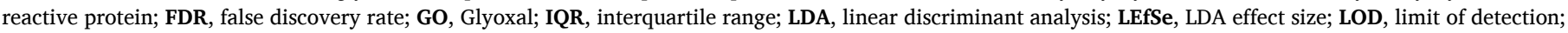

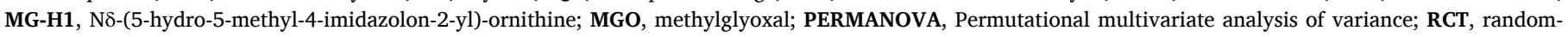
ized controlled trial; rRNA, ribosomal RNA; 3DG, 3-deoxyglucosone.

* Corresponding author at: Department of Internal Medicine, Maastricht University Medical Centre, Universiteitssingel 50, 6200MD Maastricht, the Netherlands.

E-mail addresses: katja.vandongen@wur.nl (K.C.W. van Dongen), Armand.linkens@maastrichtuniversity.nl (A.M.A. Linkens), suzan.wetzels@mumc.nl

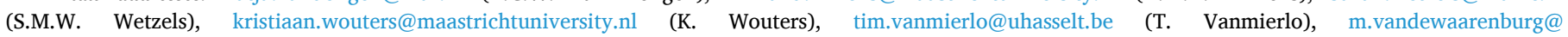

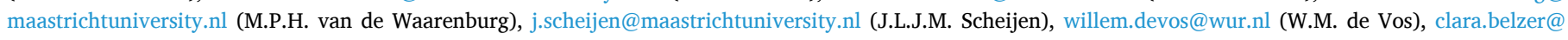
wur.nl (C. Belzer), C.Schalkwijk@maastrichtuniversity.nl (C.G. Schalkwijk).

${ }^{1}$ Katja C.W. van Dongen and Armand M.A. Linkens contributed equally to this work.

2 Clara Belzer and Casper G. Schalkwijk contributed equally to this work. 


\section{Introduction}

Advanced glycation endproducts (AGEs) are abundantly present in processed food products and may contribute to beneficial effects on flavor, smell, and shelf life (Uribarri et al., 2010). These dietary AGEs are formed during the non-enzymatic reaction between free amino acids and reducing sugars, via the so-called Maillard reaction (Maillard, 1912). There is increasing evidence in humans and animals that consumption of dietary AGEs contribute to AGEs measured in plasma (Scheijen et al., 2018; Uribarri et al., 2007) and organs (Tessier et al., 2016) and that a diet high in dietary AGEs in humans has negative biological effects, such as low-grade inflammation, endothelial dysfunction, and insulin resistance (Nowotny, Schroter, Schreiner, \& Grune, 2018). However, it is currently unknown whether AGE accumulation in tissues and the negative biological effects associated with a high AGE diet are reversible. Furthermore, how dietary AGEs are involved in the aforementioned biological effects remains poorly understood.

Dietary AGEs may exert their biological effects, at least partly, through modulation of the gut microbiota composition, which in turn is increasingly recognized to play a fundamental role in the pathophysiology of obesity, diabetes, and cardiovascular disease (Tilg \& Moschen, 2014). Although there are some discrepancies, several animal studies have shown that a heat-treated diet high in dietary AGEs can modulate gut microbiota composition (Mastrocola et al., 2020; Qu et al., 2017; Seiquer, Rubio, Peinado, Delgado-Andrade, \& Navarro, 2014; Snelson et al., 2021; Yang et al., 2020). However, the effects on inflammatory markers or increases in protein-bound and free AGE levels in plasma and/or tissues were not addressed in all of these studies. Furthermore, it is currently unknown whether changes in gut microbiota composition following a high AGE diet are reversible.

In light of the above, we hypothesized that mice fed a high dietary AGE diet for 10 weeks show increased blood and tissue AGEs, increased inflammatory markers, and different microbiota composition as compared to mice fed a standard dietary AGE diet. In addition, we studied whether changes following the high dietary AGE diet were reversible by implementing a switch after 5-weeks of high dietary AGE diet to the standard dietary AGE diet for 5 subsequent weeks.

\section{Material and methods}

\subsection{Animal studies}

To obtain a high AGE diet, a standard rodent chow (ssniff, Soest, Germany) was baked at $160{ }^{\circ} \mathrm{C}$ for two hours (hereafter referred to as "baked chow").

9-week-old female C57BL/6/OlaHsd mice were randomly divided in three cages in groups of 10 (Fig. 1). After a one-week acclimatization period, the mice were divided in three groups fed either standard chow $(\mathrm{n}=10)$, baked chow high in dietary AGEs $(\mathrm{n}=10)$, or a "switch diet" $(\mathrm{n}=10)$ for 10 weeks. In the switch diet group, mice were first fed the baked chow diet for 5 weeks, and subsequently the standard chow diet for 5 weeks. All diets were provided ad-libitum. Fecal and blood samples were collected after the 1-week acclimatization period. Blood samples were collected after 5 weeks of dietary intervention, while fecal samples were collected every 2.5 weeks. At the end of the 10-week dietary intervention, all mice were sacrificed by anesthetic overdose and plasma, liver and kidneys, and feces (from the rectum) were collected.

To test whether accumulation of AGEs in organs after a high dietary AGE diet are reversible, two extra groups of 10 mice were allocated to either 5 weeks of the standard chow diet or 5 weeks of the baked chow diet under the same conditions as mentioned above. At the end of the 5week dietary intervention, all mice were sacrificed. All experiments were approved by the local ethical committee for animal experiments of Hasselt University and performed according to institutional guidelines (matrix 201503).

\subsection{Advanced glycation end products and oxoaldehyde measurements}

AGEs were measured in chow diets, plasma, liver, and kidney. AGEs in plasma were measured as this reflects uptake of dietary AGEs from the gastrointestinal tract. We chose to measure AGEs in liver and kidney as these are major organs and are highly susceptible to AGE accumulation and AGE-induced damage (Bettiga et al., 2019; Fernando et al., 2019). Livers and kidneys were homogenized using a Mini-bead beater homogenizer (Biospec) and $250 \mu \mathrm{L}$ sodium phosphate buffer $(0.1 \mathrm{M})$ supplemented with protease inhibitor (Roche) and $0.02 \%$ Triton-x (SigmaAldrich).

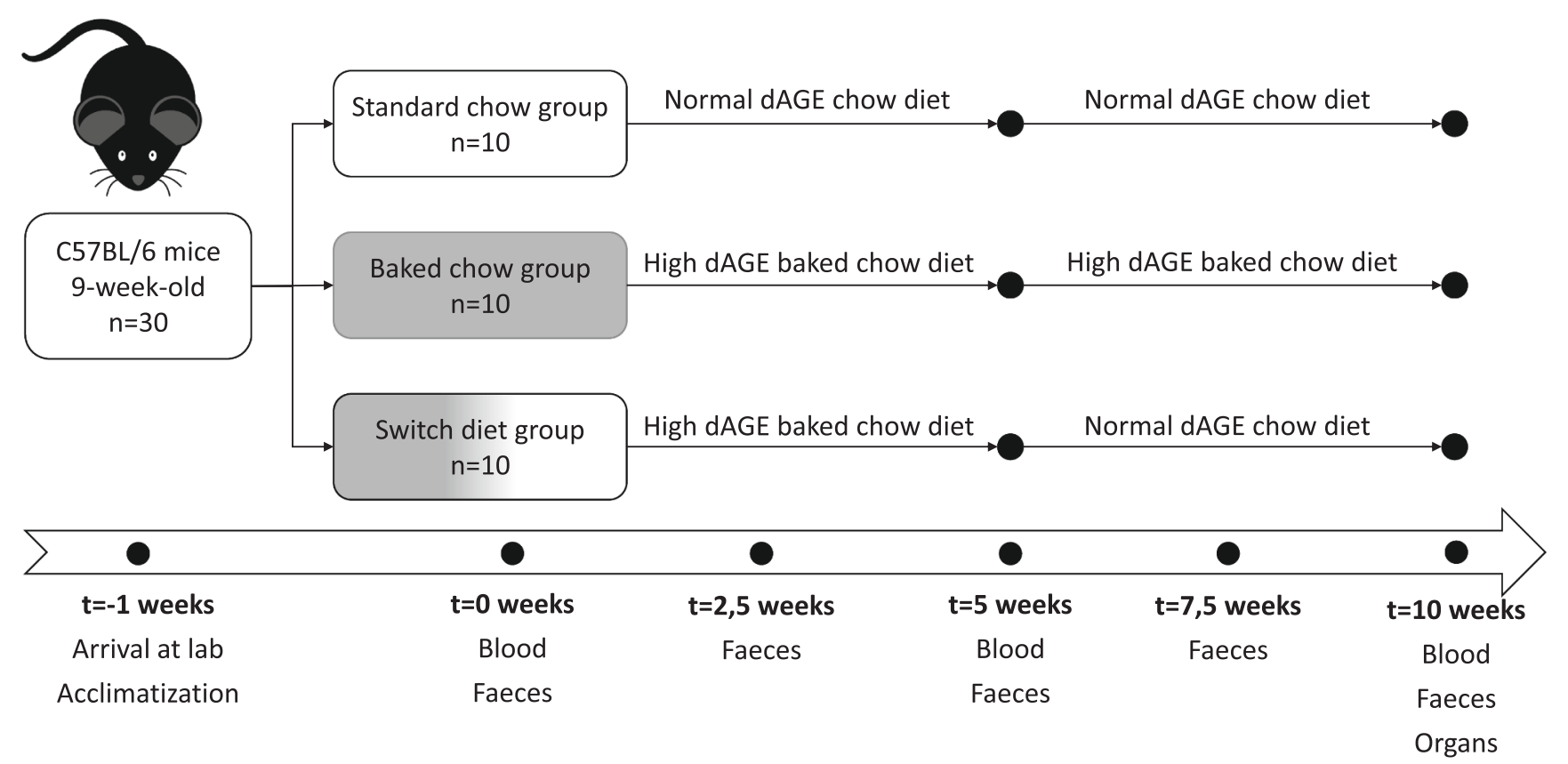

Fig. 1. Design of the main study. dAGE: dietary AGE. 
Free- and protein-bound AGEs Ne-(carboxymethyl)lysine (CML), $\mathrm{N} \varepsilon$ (1-carboxyethyl)lysine (CEL), and N8-(5-hydro-5-methyl-4-imidazolon2-yl)-ornithine (MG-H1) were analyzed in plasma, liver, and kidney homogenates by LC-MS/MS after extraction as described in detail before (Hanssen et al., 2014; Scheijen et al., 2016). Additionally, free-, proteinbound AGEs, and the oxoaldehydes methylglyoxal (MGO), glyoxal (GO) and 3-deoxyglucosone (3DG) were analysed in pulverized animal diets as described previously (Hanssen et al., 2014; Maasen et al., 2021).

\subsection{Inflammation markers}

IFN- $\gamma$, IL-10, IL-6, KC/GRO, and TNF- $\alpha$ were measured in plasma using MSD V-PLEX multiplex assay platforms (Meso Scale Diagnostics, Rockville, MD, U.S.A.). C-reactive protein (CRP) was measured using mouse CRP ELISA DuoSet kit (R\&D system, Minneapolis, Minn, U.S.A.). For IFN- $\gamma$ and IL-6, some samples were below the limit of detection (LOD) ( $<0.12 \mathrm{pg} / \mathrm{ml}$ for IFN- $\gamma, \mathrm{n}=3,<3 \mathrm{pg} / \mathrm{ml}$ for IL- $6, \mathrm{n}=14)$. These missing values were substituted by half the LOD, a commonly used method (Beal, 2001) (thus $0.06 \mathrm{pg} / \mathrm{ml}$ for IFN- $\gamma$ and $1.5 \mathrm{pg} / \mathrm{ml}$ for IL-6).

\subsection{Microbial $16 S$ rRNA sequencing}

DNA was isolated from the fecal pellets using a bead-beating procedure in combination with the customized MaxWell ${ }^{\circledR} 16$ Tissue LEV Total RNA Purification Kit (XAS1220; Promega Biotech AB, Stockholm, Sweden) as described before (Fernández-Calleja et al., 2018). Triplicate PCR reactions of the $16 \mathrm{~S}$ ribosomal RNA (rRNA) gene V4 region (515-F GTGYCAGCMGCCGCGGTAA, 806-R GGACTACNVGGGTWTCTAAT; 10 $\mu \mathrm{M}$ each) were applied to the template DNA isolates $(20 \mathrm{ng} / \mu \mathrm{L})$ with a unique barcoded sequences library approach to identify individual fecal pellets with a total volume of $35 \mu \mathrm{L}$ per reaction. Formed PCR products were qualitatively confirmed and purified as described before (Fernández-Calleja et al., 2018). $200 \mathrm{ng}$ of each sample was pooled after quantification with the Qubit $₫$ dsDNA BR Assay Kit and subsequently sequenced (Illumina NovaSeq 6000, paired-end, 150 bp; Eurofins Genomics Europe Sequencing GmbH, Konstanz, Germany).

The 16S rRNA gene sequencing raw data has been deposited in the European Nucleotide Archive (ENA) under accession number PRJEB41378.

\subsection{Microbiota data processing and analysis}

Sequences of the 16S rRNA gene were analyzed using the NG-Tax 2.0 pipeline (Poncheewin et al., 2020) with default settings, which generates de novo exact match sequence clusters: amplicon sequence variants (ASVs). ASVs with a relative abundance below $0.1 \%$ were removed and the threshold for taxonomic assignment was set at $80 \%$. The SILVA $16 \mathrm{~S}$ rRNA gene reference database release 132 (Quast et al., 2013) was used to assign taxonomy. The program $\mathrm{R}$ (version 3.6.1) was used for further data analysis. The Phyloseq package (Mcmurdie \& Holmes, 2013) (version 1.30.0) was applied to combine the ASV table with the phylogenetic tree and the metadata. ASVs with a relative abundance $>0.1 \%$ in one of the individual samples were included for further data analysis. Bray-Curtis dissimilarities (beta-diversity) were assessed with the Phyloseq package. Relative abundance composition plots at phylum and genus level were created using the Microbiome package (Lahti \& Shetty, 2017). The web-based tool Linear Discriminant Analysis (LDA) Effect Size (LEfSe) (Segata et al., 2011) was used to identify differential abundant taxa between the diet groups per sampling week. Spearman's rank correlations of microbial compositional data, glomerated at genus level, with relevant clinical data were performed with the associate function of the Microbiome package (Lahti \& Shetty, 2017) (version 1.8.0). Therefore, subsets of each sampling week were created and taxa with a relative abundance $>1 \%$ in one of the samples were included.

\subsection{Statistical analysis}

Analyses were conducted using SPSS version 25 for Windows (IBM Corporation, Armonk, NY, USA). AGE and oxoaldehyde content of standard and baked chow are presented as mean $\pm \mathrm{SD}$, and comparisons of AGE and oxoaldehyde content of standard and baked chow diets were tested using the two-tailed unpaired Students T-test.

Comparisons between free and protein-bound AGEs in plasma and tissues and inflammatory markers between groups were performed using the Mann-Whitney- $U$ test. Free and protein-bound AGEs in plasma and tissues and inflammatory markers are presented as median [IQR] due to skewed distributions.

To increase statistical efficiency and reduce multiple testing when correlating inflammatory markers to the microbiota data, an inflammatory z-score was calculated by combining TNF- $\alpha$, IFN- $\gamma$, KC/GRO, IL6 and the inverse of IL-10 (1/IL-10) as described previously (Yudkin, Stehouwer, Emeis, \& Coppack, 1999). To this end, first, z-scores for all individual parameters were calculated as follows: (individual value minus whole study population mean value)/ whole study population SD, thus resulting in a standardized variable ranging from approximately -2.5 to +2.5 SD with a mean of 0 . Second, as these individual z-scores share the same unit, they were averaged, resulting in one single inflammation score, which was subsequently standardized.

Permutational multivariate analysis of variance (PERMANOVA) of microbial beta diversity was assessed by application of the Adonis function (999 permutations) of Vegan package (Oksanen, Guillaume, Friendly, Kindt, Legendre, McGlinn, Minchin, O'Hara, Simpson, Solymos, Stevens, Szoecs, \& Wagner, 2019) (version 2.5-6). LEfSe analysis revealed differential abundant taxa if the $P$ value of the non-parametric Kruskal-Wallis test between two diet groups was $<0.05$ and the effect size of the logarithmic LDA score $>2.0$. $P$ values of correlations of microbial taxa with clinical data were adjusted for multiple testing using the Benjamini \& Hochberg false discovery rate (FDR).

All analysis were considered statistically significant with $P$ values $<$ 0.05 unless otherwise stated.

\section{Results}

\subsection{Increased levels of AGEs in chow after baking}

To assess the effect of baking on chow diets, we compared free and protein-bound AGEs, and oxoaldehyde content between standard chow and baked chow. While levels of free CEL and MG-H1 increased after the baking procedure $(34 \%, \mathrm{p}<0.05$, and $266 \%, \mathrm{p}<0.01$, respectively), free CML decreased after the baking procedure $(-54 \%, \mathrm{p}<0.05)$ (Fig. 2). In contrast, levels of protein-bound AGEs in chow were uniformly and significantly increased following the baking procedure: $299 \%$ for protein-bound CML ( $\mathrm{p}<0.001$ ), 691\% for CEL ( $\mathrm{p}<0.001$ ) and $182 \%$ for MG-H1 ( $<$ 0.001) (Fig. 2). Likewise, levels of oxoaldehydes were also significantly increased following the baking procedure: $108 \%$ for MGO (p < 0.05), 53\% for GO ( $\mathrm{p}<0.05)$ and 379\% for 3DG ( $<<0.001)$ (Fig. 2).

\subsection{Mice fed baked chow ate less than mice fed standard chow, but weight gain was similar}

To test whether weight gain over 10 weeks in young mice was different after the consumption of a baked chow diet, standard chow diet, or the switch diet, we determined food intake and weight at baseline and after 10 weeks. Mice fed the baked chow diet for 10 weeks ate less than mice fed the standard chow diet or switch diet: $2.96 \mathrm{~g} /$ day, $3.77 \mathrm{~g} /$ day, and $3.13 \mathrm{~g} /$ day, respectively (Table 1 ). In line with this, body weight was higher after 10 weeks of standard chow compared to baked chow or the switch diet, with median (g) [IQR] of 23.4 [22.2;24.1], 21.1 [20.5;21.2], and 21.8 [20.3;22.5], respectively. However, body weight of mice fed the baked chow diet was already 
A

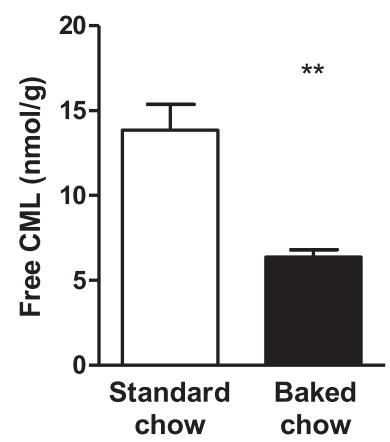

B

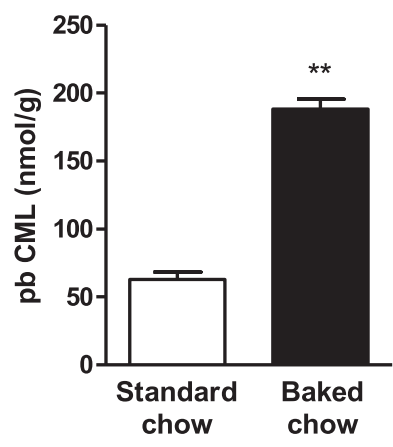

C

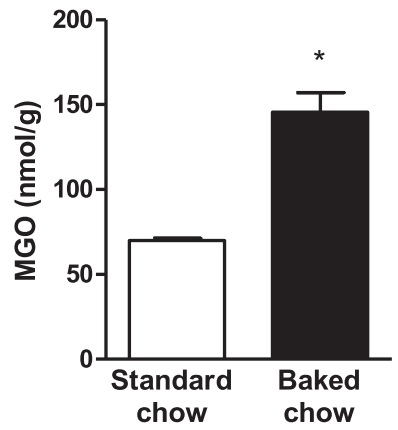

Free AGEs in chow diets

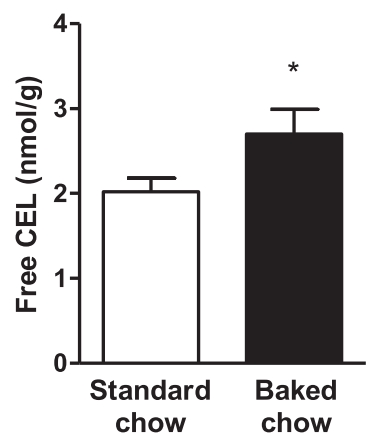

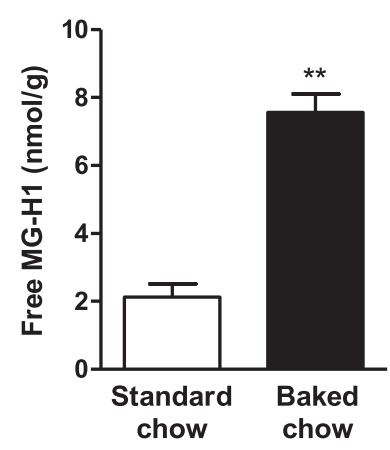

Protein-bound AGEs in chow diets

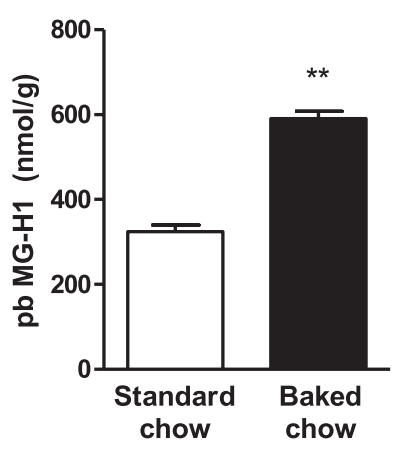

Oxoaldehydes in chow diets

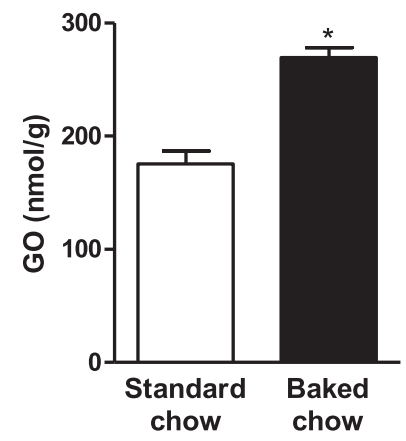

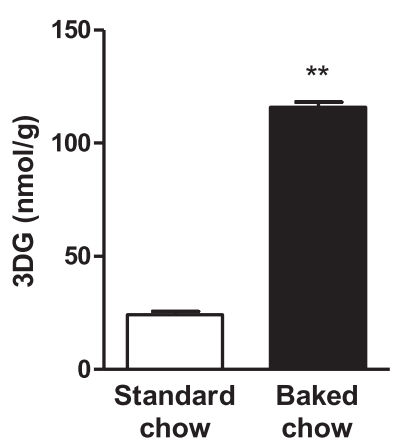

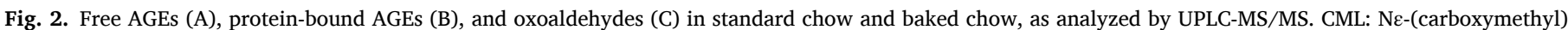

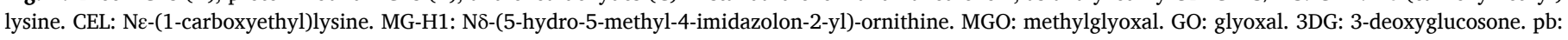

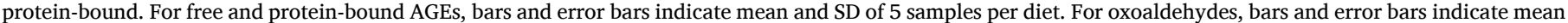

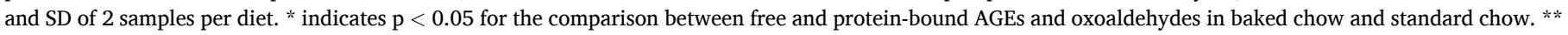
indicates $\mathrm{p}<0.001$ for the same comparison.

Table 1

Mice characteristics.

\begin{tabular}{|c|c|c|c|c|c|c|}
\hline & Standard Chow & $\begin{array}{l}\text { Baked } \\
\text { Chow }\end{array}$ & $\begin{array}{l}\text { Switch } \\
\text { Diet }\end{array}$ & $\begin{array}{l}P \\
\text { standard } \\
\text { vs baked }\end{array}$ & $\begin{array}{l}P \\
\text { standardvs switch }\end{array}$ & $\begin{array}{l}P \\
\text { Bakedvs switch }\end{array}$ \\
\hline Weight baseline (g) & 19.1 [17.9-19.5] & 18.0 [17.1-18.2] & 18.1 [16.4-19.3] & 0.019 & 0.165 & 0.481 \\
\hline Weight sacrifice (g) & $23.4[22.2-24.1]$ & $21.1[20.5-21.2]$ & 21.8 [20.3-22.5] & 0.001 & 0.004 & 0.481 \\
\hline Weight gain (g) & $4.2[3.5-5.2]$ & $3.3[2.8-3.8]$ & $3.1[2.9-4.1]$ & 0.043 & 0.035 & 0.796 \\
\hline Weight gain (\%) & 22 [19-27] & $19[15-22]$ & $17[16-23]$ & 0.218 & 0.123 & 1.000 \\
\hline Food intake (g/day) & 3.77 & 2.96 & 3.13 & & & \\
\hline
\end{tabular}

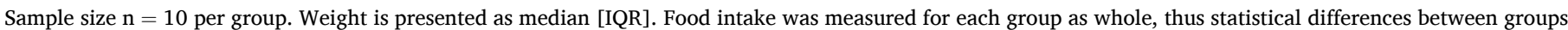
cannot be computed. Significant differences $(\mathrm{p}<0.05)$ are shown bold. 
lower at baseline as compared to mice fed the standard chow diet, thus percentage weight gain was not different between mice fed standard chow, baked chow, or the switch diet after 10 weeks, with median (\%) [IQR] of 22 [19;29], 19 [15;22], and 17 [16;23], respectively (Table 1).

\subsection{A baked chow diet increases both free and protein-bound AGEs in plasma}

To assess the impact of the chow diets on the level of AGEs after 10 weeks, we compared free and protein-bound AGEs in plasma between groups. All free AGEs in plasma were higher after baked chow compared to standard chow, with median (nmol/L) [IQR] of 536 [406;795] vs. 355 [332;430], $\mathrm{p}=0.004$ for CML, 228 [129;374] vs. 118 [86;135], $\mathrm{p}=$ 0.011 , for CEL, and 242 [89;303] vs. 76 [61;102], $p=0.019$ for MG-H1 (Fig. 3A).

Results for protein-bound AGEs in plasma were similar, except for MG-H1. After 10 weeks of the baked chow diet, protein-bound CML and CEL in plasma were higher compared to the standard chow diet, with median (nmol/L) [IQR] of 853 [805;919] vs. 561 [542;599], p $<0.001$, and 311 [227;345] vs. 207 [183;235] p = 0.011, respectively. Proteinbound MG-H1 in plasma was lower after baked chow compared to standard chow: 1464 [1377;1506] vs. 1630 [1502;1911], $\mathrm{p}=0.011$ (Fig. 3B).

\subsection{A baked chow diet increases AGEs in liver and kidneys, but mainly in the free form}

We next determined the difference in AGE levels in organs after 10 weeks of the baked chow or standard chow diet. In liver, free CML and MG-H1, but not CEL, were higher after baked chow compared to standard chow, with median (nmol/g of protein) [IQR] of 8.0 [7.2;8.5] vs. 5.4 [4.9;5.7] for CML ( $<$ < 0.001), 2.3 [1.6;3.3] vs. 0.8 [0.8;0.9] for MG$\mathrm{H} 1$ ( $\mathrm{p}<0.001)$, and $8.0[7.4 ; 8.7]$ vs. $8.1[6.8 ; 9.6]$ for CEL $(\mathrm{p}=0.730)$ (Fig. 3C). Contrarily, protein-bound AGEs in liver were not different, although there was a trend for increased protein-bound CML in liver after baked chow compared to standard chow: 21 [20;22] vs. 18 [17;21] for CML ( $\mathrm{p}=0.063), 44$ [39;46] vs. 41 [37;44] for CEL ( $\mathrm{p}=0.393)$, and $24[21 ; 28]$ vs. 25 [23;33] for MG-H1 (p=0.436) (Fig. 3D).

In kidney, free AGEs were higher after baked chow compared to standard chow, although the difference in free CML did not reach statistical significance: 224 [143;284] vs. 161 [119;179] for CML ( $\mathrm{p}=$ $0.052), 114$ [67;147] vs. 48 [37;57] for CEL (p < 0.001), and 17 [8;26] vs. $5[5 ; 6]$ for MG-H1 ( $p=0.001$ ) (Fig. 3E). In contrast, protein-bound CML in kidney, but not CEL or MG-H1, was higher in kidney after baked chow compared to standard chow: 26 [24;29] vs. 22 [19;24] for CML (p $<0.001), 19$ [18;22] vs. 21 [17;22] for CEL $(\mathrm{p}=0.796)$, and $28[22 ; 44]$ vs. $31[25 ; 36]$ for MG-H1 (p = 0.971) (Fig. $3 F$ ).

\subsection{The accumulation of AGEs in plasma and organs after a baked chow diet is reversible}

We next studied whether the accumulation of AGEs after a baked chow diet can be reversed by a standard chow diet. We first assessed whether AGEs in plasma and organs were already increased after 5 weeks of the baked chow diet. In general, all free and protein-bound AGEs were increased in plasma, kidney, and liver of mice fed the baked chow diet for 5 weeks compared to mice fed the standard chow diet for 5 weeks (Supporting information Figure S1). However, proteinbound MG-H1 in plasma was not significantly different between mice fed the baked chow diet for 5 weeks compared to the standard chow diet, with median (nmol/g of protein) [IQR] of 1599 [1487;2128] vs. 1484 [1371;1704]. In addition, free CEL was lower and protein-bound MG-H1 was not different in liver of mice fed the baked chow diet compared to the standard chow diet: $8.3[8.0 ; 8.9]$ vs. $9.6[8.8 ; 10.4], \mathrm{p}=0.007$ and $25.3[21.5 ; 28.9]$ vs. $26.5[24.9 ; 30.8], \mathrm{p}=0.280$, respectively.

The accumulation of AGEs in plasma, kidney and liver after 5 weeks of a baked chow diet were reversible, as most AGEs in plasma, liver, and kidney were significantly decreased after the switch to standard chow (Fig. 3A-F). Remarkably, free CML, free CEL, and protein-bound CEL in kidney were even lower after the switch diet as compared to 10 weeks of standard chow, with median (nmol/g of protein) [IQR] of 96 [85;102] vs. $161[119 ; 179], \mathrm{p}=0.011,31[29 ; 33]$ vs. 48 [37;57], $\mathrm{p}=0.015$, and $16[15 ; 19]$ vs. $21[17 ; 22], \mathrm{p}=0.009$, respectively (Fig. $3 \mathrm{E}$ and $3 \mathrm{~F}$ ).

\subsection{A baked chow diet affects some markers of inflammation}

To assess whether inflammation is increased after a baked chow diet compared to a standard chow diet, a panel of inflammatory markers were analyzed individually. TNF- $\alpha$, IFN- $\gamma, \mathrm{KC} / \mathrm{GRO}$, CRP, and IL- 6 were not different after 10 weeks of baked chow or standard chow (Fig. 4A). However, the anti-inflammatory cytokine IL-10 was significantly lower after baked chow compared to standard chow: $9.4 \mathrm{pg} / \mathrm{ml}[8.5 ; 10.4]$ vs. $12.8 \mathrm{pg} / \mathrm{ml}$ [10.1;14.8], $\mathrm{p}=0.011$ (Fig. 4A).

We next combined the inflammatory markers into an overall inflammatory z-score that also included IL-10. The inflammatory z-score was higher after 10 weeks of baked chow than after 10 weeks of standard chow, with median (SD) [IQR] of 0.37 [-0.13;1.31] vs. -0.39 [-1.05;0.20], $\mathrm{p}=0.029$ (Fig. 4B).

Then we determined whether the increase in inflammation after a baked chow diet could be reversed by a standard chow diet. We first assessed whether inflammatory markers in plasma were already increased after 5 weeks of the baked chow diet. In contrast to AGEs in plasma and organs, inflammatory markers in plasma and the inflammatory z-score were not already increased after 5 weeks of baked chow compared to 5 weeks of standard chow (Supporting information Figure S2). In fact, plasma TNF $\alpha$ was lower after 5 weeks of baked chow compared to 5 weeks of standard chow, with median (pg/ml) [IQR] of 5.85 [5.16-6.39] vs. 7.50 [6.48;8.42], $\mathrm{p}=0.017$.

Although inflammatory markers in plasma were not already increased after 5 weeks of baked chow, the subsequent increase in inflammatory markers after 10 weeks of baked chow could be prevented by the switch to standard chow, with inflammatory z-score median [IQR] 0.37 [-0.13;1.31] vs -0.41 [-0.81;0.26], $\mathrm{p}=0.034$ (Fig. 4B).

\subsection{Gut microbial composition is altered after feeding a baked chow diet, and is reversible}

Based on Bray-Curtis beta diversity dissimilarity distances, differences in overall microbial composition were shown in principal coordinate analysis (PCoA) plots from week 7.5 onwards (Fig. 5). Where in week 5 almost all samples were still distributed over the four quadrants (Axis $1=23.2 \%$, Axis $2=13.5 \%$ ), in week 7.5 the fecal samples from the mice fed the baked chow diet almost all clustered together in the bottom left quadrant, while almost all fecal samples from the standard chow diet clustered together in the upper right quadrant (Axis $1=23.6 \%$, Axis $2=$ $12 \%)$. The fecal samples from the mice fed the switch diet were distributed over the two upper quadrants and were located in between the samples of the baked chow and the standard chow diet. In week 10 the fecal samples of the mice fed the baked chow and the standard chow diet again were distributed over opposite quadrants, while the samples of the mice fed the switch diet were located in between these two diet groups, as expected (Axis $1=26.2 \%$, Axis $2=23 \%$ ). It should be noted that in week 10 not all fecal pellets were collected for each group $(n=6$ for the baked chow group; $n=4$ for the standard chow group; $n=2$ for the switch group).

Further PERMANOVA analysis revealed that the provided diet explained a statistically significant $(\mathrm{p}<0.001)$ variation in microbial composition from week 5 onwards with $19.5 \%$ variance explained by the diet in week 5, 22.9\% in week 7.5 and $35.2 \%$ in week 10. Bray-Curtis dissimilarities of week 0 and week 2.5 were not statistically significant. Their corresponding PCoA plots are shown in the supplementary materials (Figure S3). 
K.C.W. van Dongen et al.

Food Research International 147 (2021) 110547

A

Free AGEs in plasma

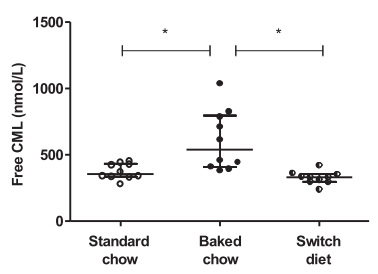

B

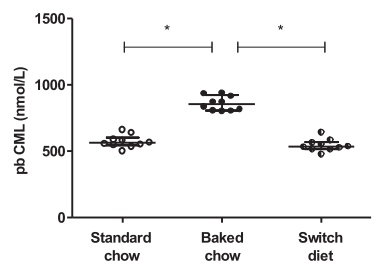

C

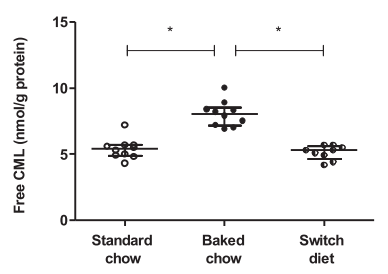

D

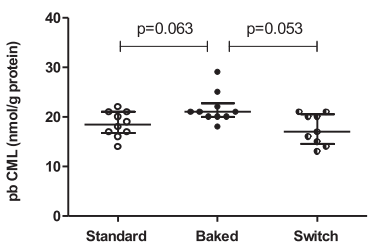$$
\text { E }
$$

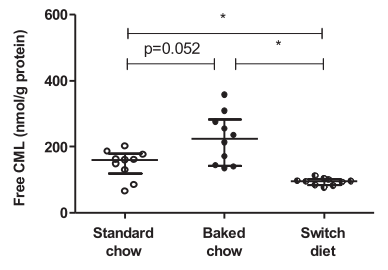

$\mathbf{F}$

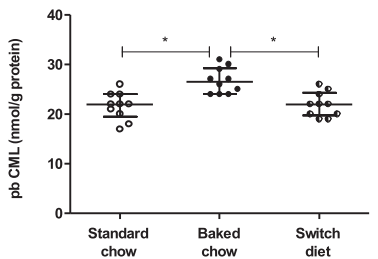

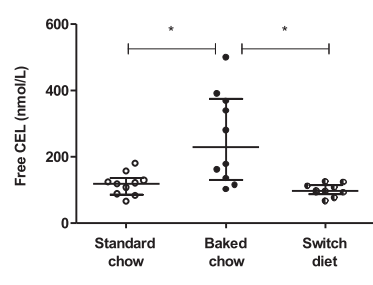

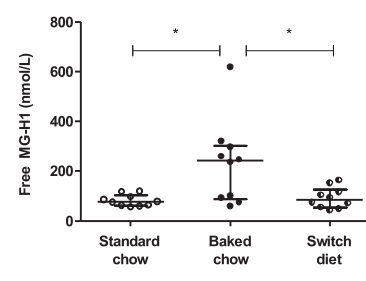

Protein-bound AGEs in plasma
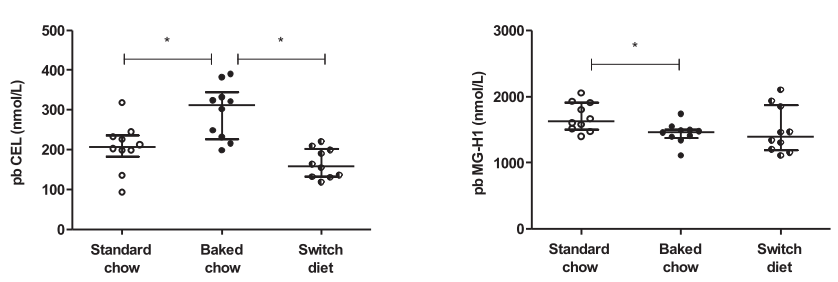

Free AGEs in liver
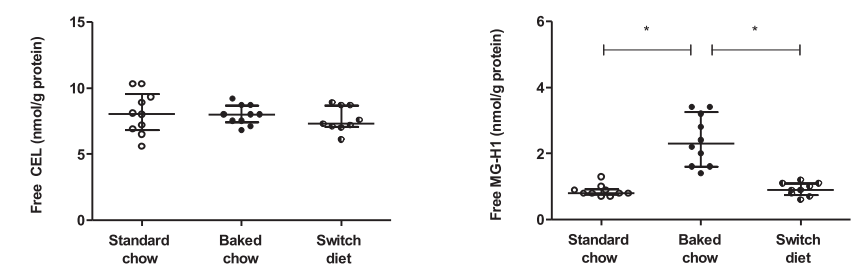

Protein-bound AGEs in liver
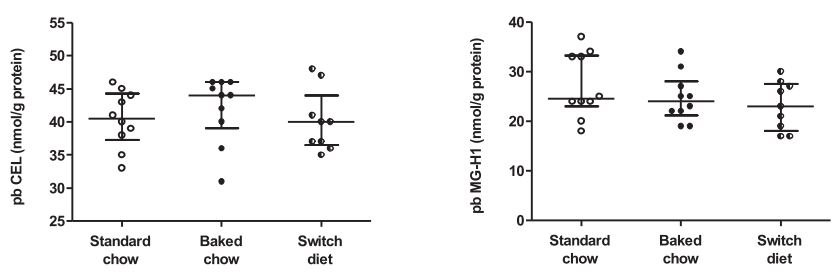

Free AGEs in kidney
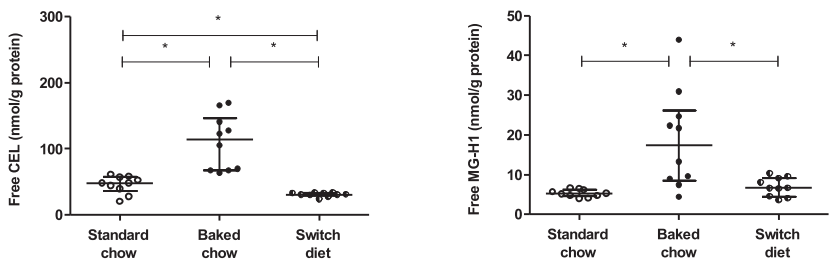

Protein-bound AGEs in kidney
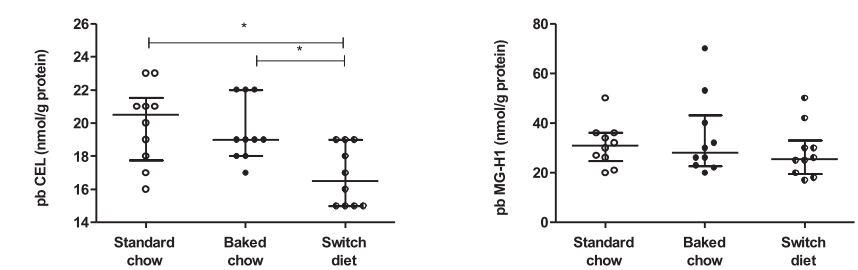

Fig. 3. Free and protein-bound AGEs in plasma (A,B), liver (C,D), and kidney (E,F) mice after 10 weeks of standard chow, baked UPLC-MS/MS. CML: Ne-(carboxymethy) lysine. CEL: Ne-(1-carboxyethyl)lysine. MGH1: N8-(5-hydro-5-methyl-4-imidazolon-2 of scatter dots indicate medina. Center ba whiskers indicate inter quartile range. * indictates $\mathrm{p}<0.05$ for the difference between
chow diets. $N=10$ for free and protein-bound AGEs in liver for the switch diet group, where $\mathrm{n}=9$. 


\section{A Individual inflammatory markers in plasma}
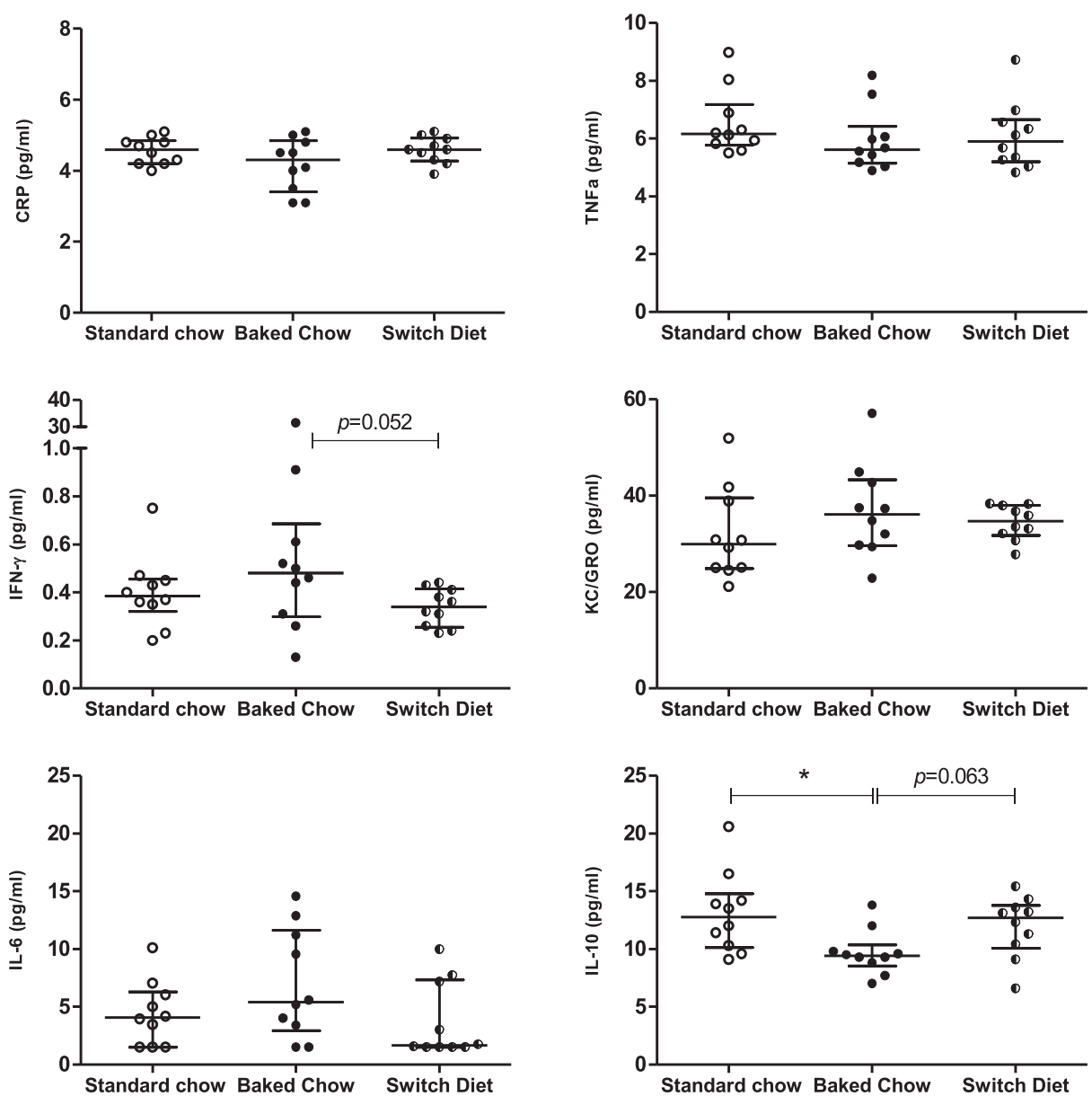

B

Inflammatory z-score

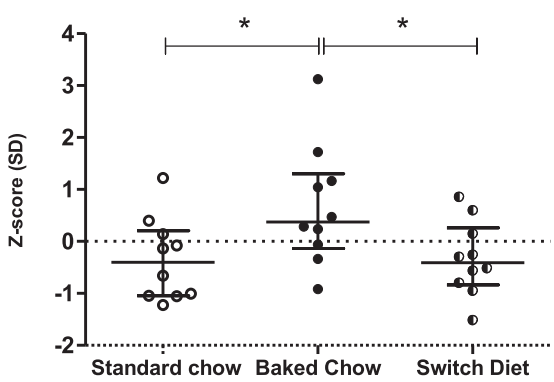

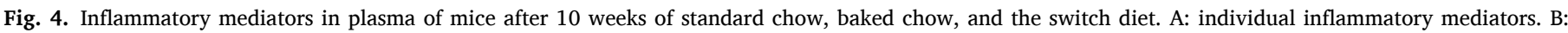

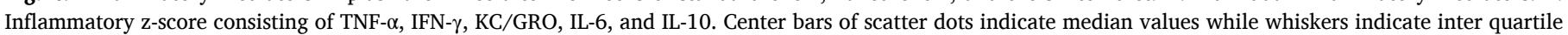
range. * indicates $\mathrm{p}<0.05$ for the difference between chow diets. $\mathrm{N}=10$ for all groups.

Relative abundance composition plots both on phylum level and genus level per sampling week showed differences between the different diet groups (Figure S4), especially at genus level. In order to identify taxa which contributed to observed differences in microbial composition between the diet groups as assessed with Bray-Curtis dissimilarities, LEfSe analysis of the microbiota composition was performed per sampling week from week 5 onwards. Differential abundant taxa were identified after comparing the microbial composition of the baked chow with the standard chow (Fig. 6) and the switch with the standard chow (Figure S5), and were visualized in cladograms (circular phylogenetic trees). Comparing the baked chow with the standard chow revealed that at week 5, six genera (i.e. Olsenella, Ruminococcaceae_UCG_009,
Dubosiella, Turicibacter, Parasutterella and Akkermansia with respective average relative abundances (\%) of 0.7, 0.1, 3.6, 0.5 and 3.2) and four families (i.e. Atopobiaceae, Erysipelotrichaceae, Burkholderiaceae, Akkermansiaceae) were enriched after the baked chow diet and three genera (i. e. Clostridiales_vadinBB60_group_uncultured, Roseburia and Faecalibaculum with respective average relative abundances (\%) of 0.1, 4.5 and 1.0) and one family (i.e. Clostridiales_vadinBB60_group) was enriched after consumption of standard chow. At week 7.5, twelve genera (i.e. Bifidobacterium, Rikenella, Eubacterium_xylanophilium_group, Lachnoclostridium, Roseburia, Ruminiclostridium_5, Ruminococcaceae_uncultured, Ruminococcaceae_UCG_009, Ruminococcaceae_UCG_014, Dubosiella, Faecalibaculum, Desulfovibrio with respective average relative abundances 

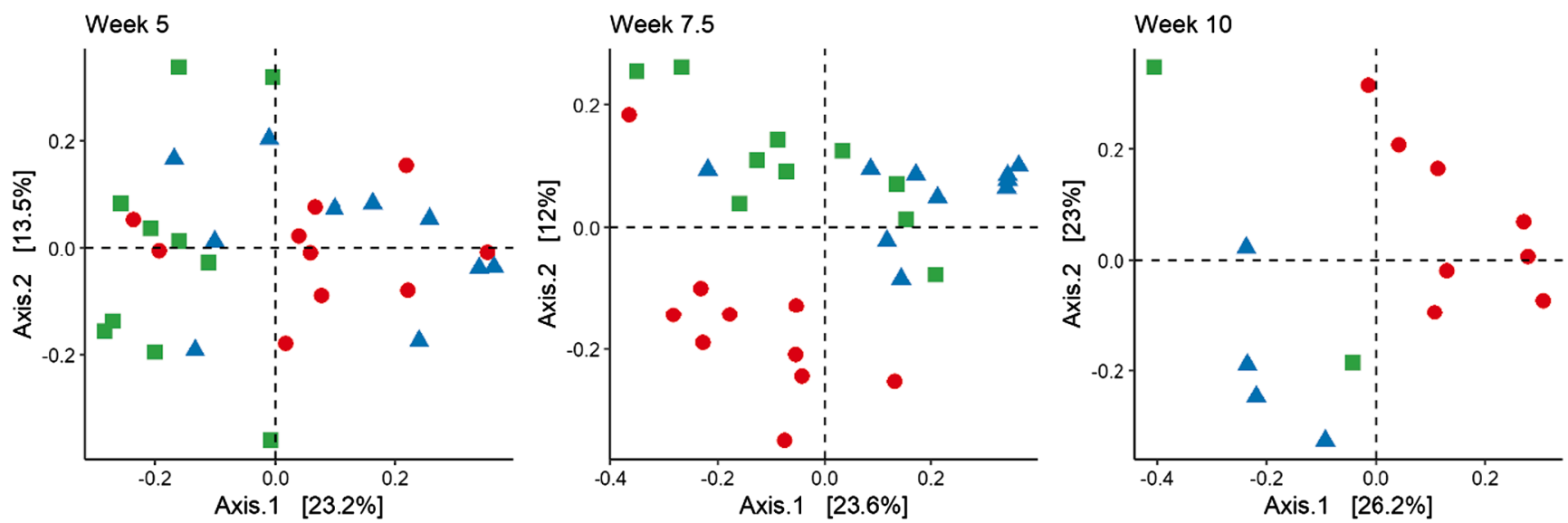

Diet Baked_chow $\Delta$ Standard_chow $\square$ Switch

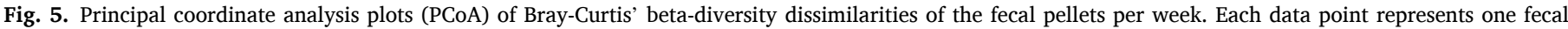

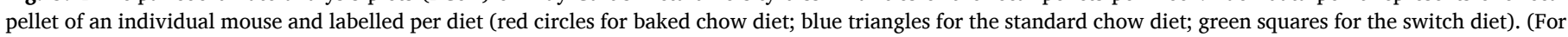
interpretation of the references to colour in this figure legend, the reader is referred to the web version of this article.)
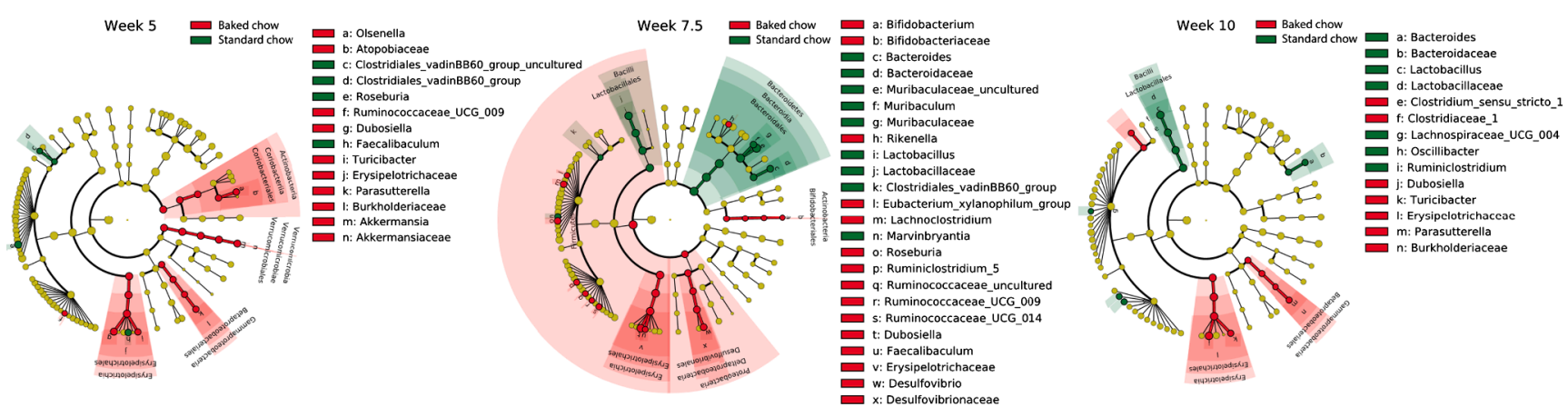

Fig. 6. LEfSe results of the significant different taxa found by comparing the mice with the baked chow diet (red) to the standard chow diet (green), sampled in week 5 ( $n=10$ per group), $7.5(n=10$ per group) and week 10 (baked chow: $n=6$; standard chow: $n=4$ ). Nomenclature was based on the highest achievable taxonomic resolution level. The alpha value was set to 0.05 and the $\log 10 \mathrm{LDA}$ score threshold to 2.0. (For interpretation of the references to colour in this figure legend, the reader is referred to the web version of this article.)

(\%) of $0.2,0.3,0.1,0.6,1.5,0.3,1.1,0.1,0.3,13.1,0.2$ and 1.7 ) and three families (i.e. Bifidobacteriaceae, Erysipelotrichaceae and Desulfovibrionaceae) were assigned as enriched taxa for the baked chow group, while for the standard chow diet five genera (i.e. Bacteroides, Muribaculaeae_uncultured, Muribaculum, Lactobacillus and Marvinbryantia with respective average relative abundances (\%) of 1.2, 46.5, 1.2, 10.5 and 0.7) and four families (i.e. Bacteroidaceae, Muribaculaceae, Lactobacillaea and Clostridiales_vadinBB60_group) were enriched. The analysis performed at week 10 identified four genera (i.e. Clostridium_sensu_stricto_1, Dubosiella, Turicibacter and Parasutterella with respective average relative abundances (\%) of 0.6, 26.2, 0.9 and 0.7) and three families (i.e. Clostridiaceae_1, Erysipelotrichaceae and Burkholderiaceae) to be enriched for the baked chow diet, while for the standard chow diet five genera (i.e. Bacteroides, Lactobacillus, Lachnospiraceae_UCG_004, Oscillibacter and Ruminiclostridium with respective average relative abundances (\%) of 1.2, 22.5, 0.1, 0.4 and 0.7) and two families (i.e. Bacteroidaceae, Lactobacillaceae) were identified to be enriched.

The microbiota of baked chow-fed mice was found to be consistently enriched in the genus Dubosiella, while that of mice fed the standard chow diet were enriched in Lactobacillus and Bacteroides from week 7.5 onwards. The highest number of identified taxa after LEfSe analysis were found in week 7.5 which could be explained by the fact that in week 10 not all fecal pellets were collected for each group ( $\mathrm{n}=6$ for the baked chow group; $\mathrm{n}=4$ for the chow group; $\mathrm{n}=2$ for the switch group), while for week 7.5 fecal pellets from all mice $(n=10$ per group) were collected and subsequently analyzed for its microbial composition.

LEfSe analysis of the switch diet with the standard chow diet (Figure S5) resulted in the highest number of enriched taxa in week 5 which decreased to week 7.5 and further to week 10. In week 10 only three genera (i.e. Dubosiella, Turicibacter and Ileibacterium with respective average relative abundances (\%) of 2.9, 3.7 and 12.4) were shown to be higher abundant in the switch diet. This indicates that the microbial composition as affected by the baked chow diet is a dietdependent reversible change.

\subsection{Gut microbiota composition data are associated with clinical parameters}

To assess whether gut microbiota composition was associated with other measured outcomes (i.e. AGE plasma and tissue levels, inflammatory markers), Spearman's rank correlations were determined after 5 and 10 weeks for mice fed the baked chow and the standard chow diet (Fig. 7). In week 5, the genera Olsenella and Turicibacter showed a statistically significant positive correlation with free plasma CML levels (Olsenella: $\rho=0.769, \mathrm{p}=0.008$; Turicibacter: $\rho=0.686, \mathrm{p}=0.030$ ) and CEL (Olsenella: $\rho=0.711, p=0.024$; Turicibacter: $\rho=0.671, p=0.031$ ). In week 10 , no bacterial taxa were statistically significantly correlated with the other outcomes after correction for multiple testing, possibly due to less fecal pellets being collected at week 10 . However, in week 10 several taxa showed a trend towards a high positive or negative 


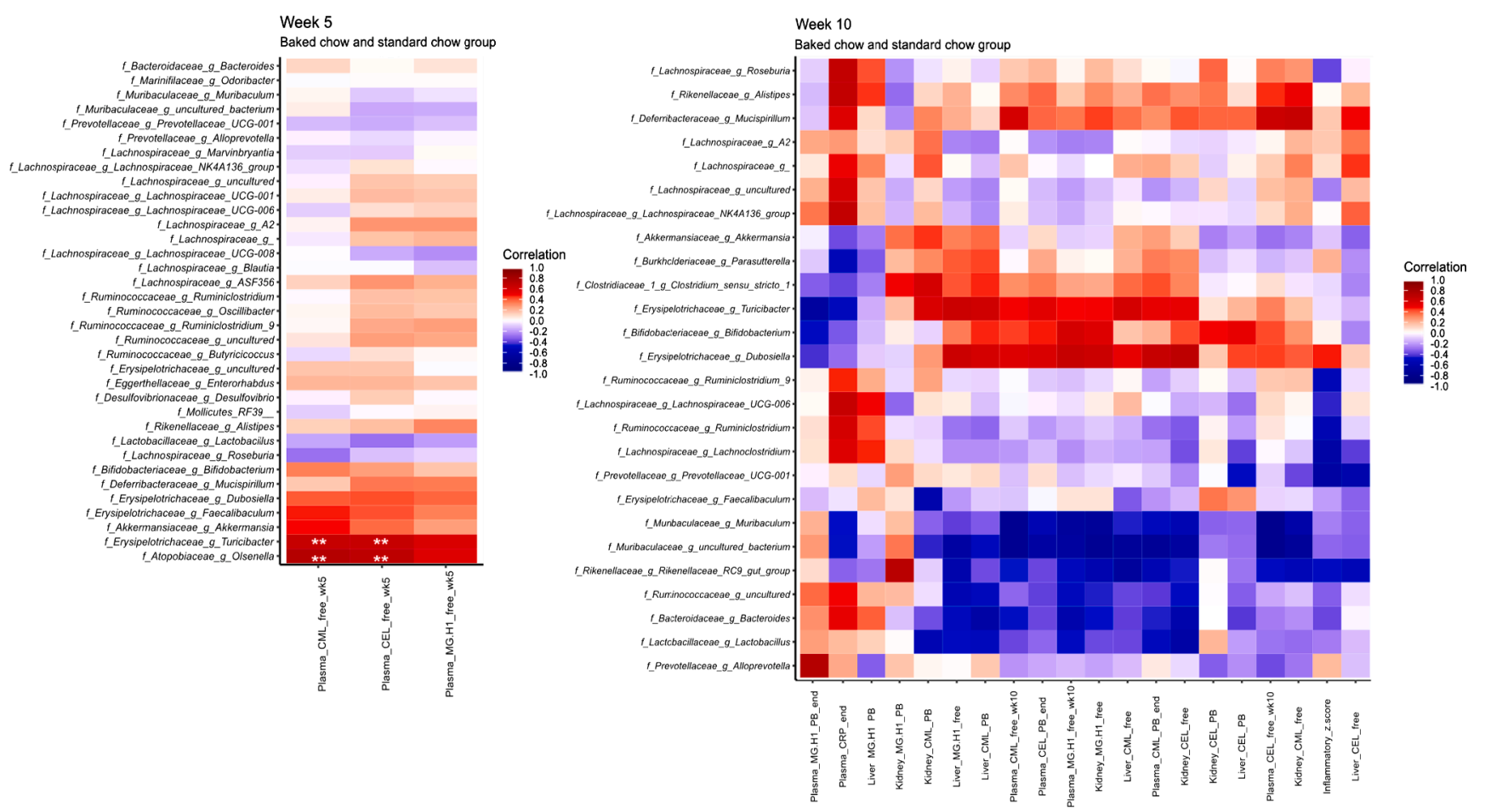

Fig. 7. Heatmaps of Spearman's rank correlation coefficient of relative abundance microbial composition data with relevant clinical parameters per sampling week, of mice in the baked chow and standard chow group. Bacterial taxa with a relative abundance $>1 \%$ in one of the samples were included. Statistically significant correlations after adjustment for multiple testing are marked with * $(\mathrm{p}<0.1)$ or $* *(p<0.05)$. Nomenclature was based on the highest achievable taxonomic resolution level. pb: protein-bound.

correlation $(\rho>0.7$ or $\rho<-0.7)$ with these outcomes. As such, Dubosiella showed a high positive correlation with free kidney CEL levels $(\rho=$ 0.719), Lachnospiraceae NK4A136 group with CRP levels in plasma ( $\rho=$ 0.709), Alloprevotella with protein-bound MG-H1 in plasma ( $\rho=0.755)$, Rikenellaceae RC9 gut group with protein-bound MG-H1 levels in the kidney ( $\rho=0.745)$ and Alistipes with plasma CRP levels $(\rho=0.709)$. On the other hand, Rikenellaceae RC9 gut group showed a high negative correlation with free CML in the liver $(\rho=-0.729)$, Muribaculaceae uncultured bacterium with free CML and CEL in plasma $(\rho=-0.797$ and $-0.825)$, protein-bound CML in plasma $(\rho=-0.755)$, free CML in the liver $(\rho=-0.753)$, and free CML and MG-H1 in the kidney $(\rho=-0.748$ and -0.783). Muribaculum showed high negative correlations with free CML and CEL in plasma, and free MG-H1 in plasma and kidney ( $\rho=$ $-0.797,-0.825,-0.718$, and -0.734 , respectively).

Since not all fecal pellets were collected at week 10 , an additional Spearman's rank correlation analysis was performed using the microbial composition data of week 7.5 with other measured outcomes determined at week 10 (Figure S6). This revealed multiple statistically significant correlations. Dubosiella showed a positive correlation with free MG-H1 in liver $(\rho=0.754, p=0.018)$, free CML in liver $(\rho=0.683$, $\mathrm{p}=0.078)$, protein-bound CML in plasma $(\rho=0.796, \mathrm{p}=0.014)$ and free CEL in kidney $(\rho=0.771, \mathrm{p}=0.014)$. Desulfovibrio showed a positive correlation with free MG-H1 levels in the liver $(\rho=0.667, p=$ 0.098). On the other hand, Muribaculum showed a negative correlation with free MG-H1 both in liver $(\rho=-0.777, \mathrm{p}=0.014)$ and kidney $(\rho=$ $-0.715, p=0.040)$. Marvinbryantia showed a negative correlation with protein-bound CEL in plasma ( $\rho=-0.744, p=0.021$ ). Although statistically significant results differed between Spearman's rank correlations using microbiome composition data from week 7.5 or week 10 , the overall trend in found correlations was comparable.

Bacteroides and Lactobacillus showed a time-dependent increase from week 5 to week 10 towards negative correlations with free and proteinbound AGE levels in plasma and tissues, while Dubosiella showed a timedependent increase towards positive correlations with free and protein- bound AGE levels in plasma and tissues. These results are in line with the taxa identified in the LEfSe analysis.

\section{Discussion}

Here, we show that consumption of a baked diet containing high levels of AGEs leads to an increase of AGEs in plasma, kidney, and liver and to a reduction of circulating IL-10 in addition to changes in gut microbiota composition in young mice. These effects were reversible or discontinued by switching the high AGE diet to a diet with lower levels of AGEs.

AGEs are generated in foods during various preparation methods involving dry heat, such as baking, grilling, frying, and toasting. Indeed, we saw a marked increase of the AGEs CML, CEL and MG-H1 after the baking of chow diet at $160{ }^{\circ} \mathrm{C}$ for two hours. Although food intake was lower in mice receiving the baked chow diet in comparison to the standard chow diet, total AGE intake was still increased in mice fed the baked chow diet. In line with this, free CML, CEL, and MG-H1 in plasma were higher in mice receiving the baked chow diet compared to mice receiving the standard chow diet. Although both free (CEL and MG-H1) and protein-bound (CML, CEL, and MG-H1) AGEs were increased in chow diets after baking, the uptake of dietary AGEs into the circulation is likely to depend mainly on digestion and absorption of protein-bound AGEs. It was previously shown that absorption of AGEs in vitro takes place mainly in the form of dipeptides, instead of free amino acid AGEs (Hellwig et al., 2011). In addition, levels of protein-bound AGEs in baked chow were approximately 30-80 fold higher than levels of free AGEs. In line with this, the increase in free plasma CML after the baked chow diet occurred in absence of an increase in free CML in baked chow.

Surprisingly, protein-bound CML and CEL in plasma were also higher, while protein-bound MG-H1 was lower in mice receiving the baked chow diet compared to mice receiving the standard chow diet. As such, we would not expect a direct increase in plasma protein-bound AGEs following the high AGE baked chow diet. This would be in line 
with our previous data in humans showing that the free form of AGEs in plasma, but not the protein-bound form, is associated with dietary AGE intake in participants of the CODAM study (Scheijen et al., 2018). However, randomized controlled trials (RCTs) in humans are still inconclusive as there are reports of both an increase in protein-bound AGEs in plasma (Birlouez-Aragon et al., 2010) and no change (de Courten et al., 2016) after a high-AGE diet. This distinction is important, as the origin of both forms of AGEs is different, and it has implications for their biological effects, for example as free AGEs do not have affinity for the receptor for AGEs (RAGE) (Kislinger et al., 1999; Penfold et al., 2010; Xie et al., 2008).

We also observed some surprising findings in organs of mice fed the baked chow diet. While we observed higher levels of most free AGEs in liver and kidney in these mice, we also observed higher protein-bound CML in kidney. Direct evidence for dietary AGE accumulation in organs is already available in animal models, but free- and protein-bound AGEs have not been assessed simultaneously (Elmhiri et al., 2015; Roncero-Ramos et al., 2013; Somoza et al., 2006; Tessier et al., 2016). Tessier et al. used ${ }^{13} \mathrm{C}$-labeled CML to discriminate between dietary and endogenous CML and found that dietary CML accumulated in several organs of mice, but they could not discriminate between free and protein-bound CML due to low quantities of tissue being available (Tessier et al., 2016). The increase in protein-bound CML and CEL in plasma and protein-bound CML in kidney of mice fed the baked chow diet in the present study could be the result from indirect effects of dietary AGEs. It has previously been shown that dietary CML and MGOmodified albumin lead to significant increases of NF-KB and RAGE in kidneys of piglets and white adipose tissue respectively (Cai et al., 2012; Elmhiri et al., 2015). As such, protein-bound AGEs in plasma and tissues may reflect increased endogenous formation of AGEs, and not a direct uptake of dietary AGEs. In addition, we also measured reactive precursors of AGEs in the chow diet, which were increased upon heating. The increased levels of these reactive oxoaldehydes in baked chow diet may give rise to increased endogenous levels of protein-bound AGEs.

In line with the increase in AGEs in plasma and tissue, we observed reduced levels of circulating IL-10 in mice fed the baked chow diet as compared to mice fed the standard chow diet. Also driven by this, the inflammatory z-score was significantly higher after the baked chow diet compared to the standard chow diet. In line with our findings, Rajan et al. observed a significant increase in expression of several proinflammatory cytokines in 12-week old mice after a 6-month dietary intervention with baked chow diet (Sowndhar Rajan et al., 2018) and Mastrocola et al. also observed a decrease in IL-10 after feeding a high AGE diet for 22 weeks in 4-week old mice (Mastrocola et al., 2020). Although with the current data we cannot provide insights in the origin of increased inflammation following the baked chow diet, the increase in protein-bound CML in kidney is suggestive of involvement of the kidneys. Future studies could provide insight in a potential direct link between increased protein-bound levels in a specific tissue with the origin of observed inflammation. Nonetheless, the increase in inflammation after the baked chow diet may provide some insight into the mechanisms behind the biological effects of dietary AGEs. Circulating IL-10 levels are lower in obese insulin resistant individuals and treatment with IL-10 improves lipid-induced insulin resistance (Hong et al., 2009). As such, the decrease in circulating IL-10 after the baked chow diet could potentially contribute to the decrease in insulin sensitivity observed in humans after a high-AGE diet (de Courten et al., 2016). However, in humans, the increase in inflammation after a high-AGE diet is observed in some RCTs (Baye, Kiriakova, et al., 2017) but not all (Baye, De Courten, et al., 2017) and deserves further investigation.

To our knowledge, we are the first to show that the accumulation of AGEs in plasma and organs after a diet high in dietary AGEs is reversible. We showed that most AGEs in plasma, kidney, and liver were already increased after 5 weeks of the baked chow diet and that this accumulation of AGEs is fully reversible, by switching the diet to standard chow for 5 subsequent weeks. Although reversibility may be expected for
AGEs in plasma, as they are rapidly cleared by the kidneys (Förster \& Henle, 2003), the latter does not necessarily apply to dietary AGEs accumulating in organs. We can currently only speculate on how dietary AGEs are transported into organs, if they end up in the intra- or extracellular matrix, and if glycated proteins (i.e. protein-bound AGEs) show different protein turnover rates. For example, the extracellular space is less subjected to protein turnover (Kerrigan, Mansell, \& Sandy, 2000). Additionally, glycated proteins in food show resistance to enzymatic breakdown in the gastrointestinal tract (Pinto et al., 2014) and this could also potentially apply to enzymatic protein turnover. Inflammation, on the other hand, was not already increased after 5 weeks of the baked chow diet. Although mice fed the switch diet showed decreased inflammation compared to mice fed the baked chow diet for 10 weeks, we cannot speak of true reversibility. Nonetheless, dietary AGEs have been associated with negative biological effects in humans (Nowotny et al., 2018), but also several negative clinical outcomes, such as insulin resistance (de Courten et al., 2016), weight gain (Cordova et al., 2019), and vertebral fractures (Waqas et al., 2020). Therefore, the important finding of reversibility of AGE accumulation in plasma and organs may have beneficial implications for those whom ingest a diet high in AGEs to lower the dietary AGE-associated negative biological effects and clinical outcomes. Furthermore, the observation of reversible AGE accumulation after modulation of dietary AGEs also further strengthens their causal link.

Next to effects of baked chow diets on AGE levels in plasma and tissues and inflammatory markers, the baked chow diet also altered the gut microbiota composition of mice compared to the standard chow diet. From week 5 onwards significant effects of the baked chow diet on the gut microbiota composition were observed by assessing Bray-Curtis dissimilarities. Interestingly, statistically significant effects on gut microbiota composition were earlier observed (i.e. week 5) compared to effects on the inflammatory z-score and IL-10 levels in plasma (i.e. week 10). In the baked chow group, the genus Dubosiella was consistent enriched. Dubosiella was recently isolated from mice (Cox, Sohn, Tyrrell, Citron, Lawson, Pate, Iizumi, Perez-Perez, Goldstein, \& Blaser, 2017), and has not been associated with specific functions before. However, Dubosiella belongs to the family Erysipelotrichaceae and was found to be related to Allobaculum stercoricanis (Cox et al., 2017). The genus Allobaculum spp. was increased in relative abundance in two animal studies after a glycated or high AGE diet (Han et al., 2018; Qu et al., 2017), which might imply a specific genera-cluster or family as a potential target for a high AGE diet. Interestingly, this does not corroborate with previous findings by Yang et al. (2020) who found an enrichment in the order Erysipelotrichales after the control diet instead of the high AGE diet. However, no specific genus was identified and it is relevant to note that old mice (15-month-old) were studied (Yang et al., 2020).

On the other hand, Lactobacillus and Bacteroides were consistent enriched in the standard chow group, corresponding to a relative decrease in the baked chow group, which corroborates partially or fully with earlier findings showing a contraction in Lactobacillus spp. or Bacteroides spp. (Delgado-Andrade et al., 2017; Mastrocola et al., 2020; Qu et al., 2017, 2018; Seiquer et al., 2014; Snelson et al., 2021). However, not all animal studies found this contraction in Lactobacillus spp. or Bacteroides spp. (Yang et al., 2020), indicating the need for multiple studies to derive clear and consistent conclusions regarding effects on gut microbiota composition.

In week 5, the genera Olsenella and Turicibacter showed a positive statistically significant correlation with free CML and CEL in plasma, while in week 10 no statistically significant correlations were found after correction for multiple testing. This could be explained by the higher number of parameters involved in the correlations of week 10 compared to week 5. Overall, Dubosiella showed high correlations with most parameters measured at week 10 , and was included in statistical significant positive correlations when applying microbiome composition data from week 7.5 instead of week 10 .

Our data suggests that Dubosiella spp. are associated with a diet high 
in AGE levels. However, further work is required to identify the function or role of Dubosiella spp. in order to explain this link with AGEs. A hypothesized explanation of the enrichment of Dubosiella spp. in the baked chow diet group is the utilization of AGEs as a substrate by the bacterium. Multiple bacterial strains were reported in literature to degrade CML. For example Cloacibacillus evryvenis (Bui, Troise, Fogliano, \& De Vos, 2019), isolated from human feces, and Escherichia coli strains (Hellwig, Auerbach, Mü, Samuel, Kammann, Beer, Gunzer, \& Henle, 2019) were shown to degrade CML in vitro, which proves the ability of specific gut bacteria to utilize an AGE.

An attempt was made to investigate whether the effects of the baked chow diet on gut microbial composition were reversible by including the switch diet group. The results of both the Bray-Curtis dissimilarities and LEfSe analysis indicated that the trend of changing microbiota composition due to the baked chow diet is reversible. In week 10, LEfSe analysis identified 3 genera (i.e. Dubosiella, Turicibacter and Ileibacterium) to be enriched in the switch diet group. These findings suggest that minor differences in microbiota composition between the standard chow and the switch diet were still observed, which can be explained by the fact that the gut microbiota requires time to adapt towards a new diet composition. It could be that a 5-week dietary intervention of the standard chow diet after 5 weeks of the baked chow diet was too short to completely adapt and consequently forming a comparable microbiota composition as in the 10-week standard chow diet group.

Our study has several strengths. Protein-bound and free AGEs were measured both in the chow diets as well as in plasma and organs of mice with the highly specific gold standard UPLC-MS/MS technique. To our knowledge, we are the first to concurrently measure both forms of AGEs in both the experimental diet and tissue compartments. In addition, the validity of our findings is increased by inclusion of a control group and the switch group. Also, inclusion of the switch group enables us to show that changes in AGE accumulation following a high AGE diet are reversible in nature. Moreover, it is important to highlight that the chow baking conditions were not substantially different from the conditions used in other studies, and while AGE levels in the baked chow diet were increased, they still represented realistic dietary levels. Finally, we used very young, healthy mice without any underlying disease.

Our study also has several limitations. Primarily, we cannot rule out that the baking procedure has had effects on chow other than increasing AGEs, such as decreasing vitamin bioavailability or increasing acrylamide formation. Additionally, mice weighed less at the end of the study after the baked chow diet compared to the standard chow diet, and food intake was lower of mice in the baked chow diet group. However, percentual increase of weight did not differ between groups, and in addition bacterial taxa associated with weight loss, such as Allobaculum (Ravussin et al., 2012) or Akkermansia muciniphila (Everard et al., 2013), were not showing a similar trend towards lower weight in the baked chow diet group. If anything, lower food intake in the baked chow group reduced exposure to dietary AGEs and lead to underestimations of their effects. Finally, our observations of reversible AGE accumulation in kidney and liver may not be extrapolated to other organs. Likewise, our findings in mice cannot be directly extrapolated to humans, as species differences exist in for example metabolic rate and dietary habits, as also in gut microbiota composition (Hugenholtz \& de Vos, 2018).

In summary, intake of dietary AGEs results in reversible elevated levels of AGEs in plasma and organs and alterations in the gut microbiota composition with a change in the inflammatory profile of healthy young mice. Randomized controlled trials on the effects of dietary AGEs on gut microbiota in humans are needed.

\section{Funding}

This work was partially supported by the SIAM Gravitation Grant 024.002.002 and 2008 Spinoza Award of the Netherlands Organization for Scientific Research to WMdV.

\section{CRediT authorship contribution statement}

Katja C.W. Dongen: Formal analysis, Writing - original draft, Writing - review \& editing, Visualization. Armand M.A. Linkens: Formal analysis, Writing - original draft, Writing - review \& editing, Visualization. Suzan M.W. Wetzels: Investigation, Writing - review \& editing, Project administration. Kristiaan Wouters: Conceptualization, Investigation. Tim Vanmierlo: Conceptualization, Investigation, Resources, Funding acquisition. Marjo P.H. Waarenburg: Methodology, Validation, Formal analysis. Jean L.J.M. Scheijen: Methodology, Validation, Formal analysis. Willem M. Vos: Resources, Writing - review \& editing, Supervision, Funding acquisition. Clara Belzer: Resources, Writing - review \& editing, Supervision, Funding acquisition. Casper G. Schalkwijk: Conceptualization, Resources, Writing - review \& editing, Supervision, Funding acquisition.

\section{Declaration of Competing Interest}

The authors declare that they have no known competing financial interests or personal relationships that could have appeared to influence the work reported in this paper.

\section{Acknowledgements}

We thank Diabetesfonds, ZonMw and Graduate school VLAG for their financial support.

\section{Appendix A. Supplementary material}

Supplementary data to this article can be found online at https://doi. org/10.1016/j.foodres.2021.110547.

\section{References}

Baye, E., De Courten, M. P. J., Walker, K., Ranasinha, S., Earnest, A., Forbes, J. M., et al. (2017). Effect of dietary advanced glycation end products on inflammation and cardiovascular risks in healthy overweight adults: A randomised crossover trial. Scientific Reports, 7(1), 1-6. https://doi.org/10.1038/s41598-017-04214-6.

Baye, E., Kiriakova, V., Uribarri, J., Moran, L. J., \& De Courten, B. (2017). Consumption of diets with low advanced glycation end products improves cardiometabolic parameters: Meta-analysis of randomised controlled trials. Scientific Reports, 7(1), 1-9. https://doi.org/10.1038/s41598-017-02268-0.

Beal, S. L. (2001). Ways to fit a PK model with some data below the quantification limit. Journal of Pharmacokinetics and Pharmacodynamics, 28(5), 481-504. https://doi.org/ 10.1023/A:1012299115260.

Bettiga, A., Fiorio, F., Di Marco, F., Trevisani, F., Romani, A., Porrini, E., et al. (2019). The modern western diet rich in advanced glycation end-Products (AGEs): an overview of its impact on obesity and early progression of renal pathology. Nutrients, 11(8), 1748. https://doi.org/10.3390/nu11081748.

Birlouez-Aragon, I., Saavedra, G., Tessier, F. J., Galinier, A., Ait-Ameur, L., Lacoste, F., et al. (2010). A diet based on high-heat-treated foods promotes risk factors for diabetes mellitus and cardiovascular diseases. American Journal of Clinical Nutrition, 91(5), 1220-1226. https://doi.org/10.3945/ajcn.2009.28737.

Bui, T. P. N., Troise, A. D., Fogliano, V., \& De Vos, W. M. (2019). Anaerobic degradation of $\mathrm{N}$ - $\epsilon$-carboxymethyllysine, a major glycation end-product, by human intestinal bacteria. Journal of Agricultural and Food Chemistry, 67(23), 6594-6602. https://doi. org/10.1021/acs.jafc.9b02208.

Cai, W., Ramdas, M., Zhu, L., Chen, X., Striker, G. E., \& Vlassara, H. (2012). Oral advanced glycation endproducts (AGEs) promote insulin resistance and diabetes by depleting the antioxidant defenses AGE receptor-1 and sirtuin 1. Proc Natl Acad Sci U S A, 109(39), 15888-15893. https://doi.org/10.1073/pnas.1205847109.

Cordova, R., Knaze, V., Viallon, V., Rust, P., Schalkwijk, C. G., Weiderpass, E., et al. (2019). Dietary intake of advanced glycation end products (AGEs) and changes in body weight in European adults. European Journal of Nutrition. https://doi.org/ 10.1007/s00394-019-02129-8.

Cox, L. M., Sohn, J., Tyrrell, K. L., Citron, D. M., Lawson, P. A., Pate, N. B., Iizumi, T., Perez-Perez, G. I., Goldstein, E. J. C., \& Blaser, M. J. (2017). Description of two novel members of the family erysipelotrichaceae: Ileibacterium valens gen. nov., sp. nov. and Dubosiella newyorkensis, gen. nov., sp. nov., from the murine intestine, and emendation to the description of Faecalibacterium rodentium. International Journal of Systematic and Evolutionary Microbiology, 67(5), 1247-1254. https://doi.org/ 10.1099/ijsem.0.001793.

de Courten, B., de Courten, M. P., Soldatos, G., Dougherty, S. L., Straznicky, N., Schlaich, M., et al. (2016). Diet low in advanced glycation end products increases insulin sensitivity in healthy overweight individuals: A double-blind, randomized, 
crossover trial. The American Journal of Clinical Nutrition, 103(6), 1426-1433. https://doi.org/10.3945/ajcn.115.125427.

Delgado-Andrade, C., Pastoriza de la Cueva, S., Peinado, M. J., Rufián-Henares, J.Á., Navarro, M. P., \& Rubio, L. A. (2017). Modifications in bacterial groups and short chain fatty acid production in the gut of healthy adult rats after long-term consumption of dietary Maillard reaction products. Food Research International, 100, 134-142. https://doi.org/10.1016/j.foodres.2017.06.067.

Elmhiri, G., Mahmood, D. F., Niquet-Leridon, C., Jacolot, P., Firmin, S., Guigand, L., et al. (2015). Formula-derived advanced glycation end products are involved in the development of long-term inflammation and oxidative stress in kidney of IUGR piglets. Molecular Nutrition \& Food Research, 59(5), 939-947. https://doi.org/ 10.1002/mnfr.201400722.

Everard, A., Belzer, C., Geurts, L., Ouwerkerk, J. P., Druart, C., Bindels, L. B., et al. (2013). Cross-talk between Akkermansia muciniphila and intestinal epithelium controls diet-induced obesity. Proceedings of the National Academy of Sciences of the United States of America, 110(22), 9066-9071. https://doi.org/10.1073/ pnas.1219451110.

Fernández-Calleja, J. M. S., Konstanti, P., Swarts, H. J. M., Bouwman, L. M. S., GarciaCampayo, V., Billecke, N., et al. (2018). Non-invasive continuous real-time in vivo analysis of microbial hydrogen production shows adaptation to fermentable carbohydrates in mice. Scientific Reports, 8(1), 1-16. https://doi.org/10.1038/ s41598-018-33619-0.

Fernando, D. H., Forbes, J. M., Angus, P. W., \& Herath, C. B. (2019). Development and progression of non-alcoholic fatty liver disease: The role of advanced glycation end products. In International Journal of Molecular Sciences (Vol. 20, Issue 20). MDPI AG. https://doi.org/10.3390/ijms20205037.

Förster, A., \& Henle, T. (2003). Glycation in food and metabolic transit of dietary AGEs (advanced glycation end-products): studies on the urinary excretion of pyrraline. Biochemical Society Transactions, 31(6), 1383-1385. http://portlandpress.com/ biochemsoctrans/article-pdf/31/6/1383/537657/bst0311383.pdf?casa token $=$ R0 Xb0E0xu0AAAAA:0v3WhTZtzCkTSSMgqrEg0X9LW1EiPWMqcWjeYFygvHKVMR7S o1fPWfhdgwRWRV_ikVerTZsBZw.

Han, K., Jin, W., Mao, Z., Dong, S., Zhang, Q., Yang, Y., et al. (2018). Microbiome and butyrate production are altered in the gut of rats fed a glycated fish protein diet. Journal of Functional Foods, 47, 423-433. https://doi.org/10.1016/j.jff.2018.06.007.

Hanssen, N. M., Wouters, K., Huijberts, M. S., Gijbels, M. J., Sluimer, J. C., Scheijen, J. L. et al. (2014). Higher levels of advanced glycation endproducts in human carotid atherosclerotic plaques are associated with a rupture-prone phenotype. European Heart Journal, 35(17), 1137-1146. https://doi.org/10.1093/eurheartj/eht402.

Hellwig, M., Auerbach, C., Mü, N., Samuel, P., Kammann, S., Beer, F., Gunzer, F., \& Henle, T. (2019). Metabolization of the Advanced Glycation End Product N$\varepsilon$-Carboxymethyllysine (CML) by Different Probiotic E. coli Strains. https://doi.org/ 10.1021/acs.jafc.8b06748.

Hellwig, M., Geissler, S., Matthes, R., Peto, A., Silow, C., Brandsch, M., et al. (2011) Transport of free and peptide-bound glycated amino acids: Synthesis, transepithelial flux at Caco-2 cell monolayers, and interaction with apical membrane transport proteins. ChemBioChem, 12(8), 1270-1279. https://doi.org/10.1002/ cbic. 201000759.

Hong, E. G., Hwi, J. K., Cho, Y. R., Kim, H. J., Ma, Z., Yu, T. Y., et al. (2009). Interleukin10 prevents diet-induced insulin resistance by attenuating macrophage and cytokine response in skeletal muscle. Diabetes, 58(11), 2525-2535. https://doi.org/10.2337/ db08-1261.

Hugenholtz, F., \& de Vos, W. M. (2018). Mouse models for human intestinal microbiota research: a critical evaluation (pp. 149-160). Birkhauser Verlag AG. https://doi.org/ 10.1007/s00018-017-2693-8.

Kerrigan, J. J., Mansell, J. P., \& Sandy, J. R. (2000). Matrix turnover. In Journal of Orthodontics (Vol. 27, Issue 3, pp. 227-233). Maney Publishing. https://doi.org/ 10.1179/ortho.27.3.227

Kislinger, T., Fu, C., Huber, B., Qu, W., Taguchi, A., Yan, S. Du., et al. (1999). N (ع)-(carboxymethyl)lysine adducts of proteins are ligands for receptor for advanced glycation end products that activate cell signaling pathways and modulate gene expression. Journal of Biological Chemistry, 274(44), 31740-31749. https://doi.org/ 10.1074/jbc. 274.44.31740.

Lahti, L., Shetty, S. A., \& et al. (2017). Tools for microbiome analysis in R. Http:// Microbiome.Github.Com/Microbiome. http://microbiome.github.com/microbiome.

Maasen, K., Scheijen, J. L. J. M., Opperhuizen, A., Stehouwer, C. D. A., Van Greevenbroek, M. M., \& Schalkwijk, C. G. (2021). Quantification of dicarbonyl compounds in commonly consumed foods and drinks; presentation of a food composition database for dicarbonyls. Food Chemistry, 339, Article 128063. https:// doi.org/10.1016/j.foodchem.2020.128063.

Maillard, L. C. (1912). Action des acides amines sur les sucres; formation des melanoidines par voie methodique. Comptes R. Acad. Sci. (Paris), 154, 66-68. https://ci.nii.ac.jp/naid/10005396269/en/.

Mastrocola, R., Collotta, D., Gaudioso, G., Le Berre, M., Cento, A. S., Ferreira Alves, G., et al. (2020). Effects of exogenous dietary advanced glycation end products on the cross-talk mechanisms linking microbiota to metabolic inflammation. Nutrients, 12 (9), 2497. https://doi.org/10.3390/nu12092497.

Mcmurdie, P. J., \& Holmes, S. (2013). phyloseq: An R package for reproducible interactive analysis and graphics of microbiome census data. PLOS ONE, 8(4), Article e61217. https://doi.org/10.1371/journal.pone.0061217.

Nowotny, K., Schroter, D., Schreiner, M., \& Grune, T. (2018). Dietary advanced glycation end products and their relevance for human health. Ageing Res Rev, 47, 55-66. https://doi.org/10.1016/j.arr.2018.06.005.

Oksanen, J., Guillaume, B., Friendly, M., Kindt, R., Legendre, P., McGlinn, D., Minchin, P. R., O'Hara, R. B., Simpson, G. L., Solymos, P., Stevens, M. H. H., Szoecs, E., \& Wagner, H. (2019). vegan: Community Ecology Package. R package version 2.5-6.
Https://Cran.r-Project.Org/Package=vegan. https://cran.r-project.org/ package $=$ vegan .

Penfold, S. A., Coughlan, M. T., Patel, S. K., Srivastava, P. M., Sourris, K. C., Steer, D., et al. (2010). Circulating high-molecular-weight RAGE ligands activate pathways implicated in the development of diabetic nephropathy. Kidney International, 78(3), 287-295. https://doi.org/10.1038/ki.2010.134.

Pinto, M. S., Léonil, J., Henry, G., Cauty, C., Carvalho, A.Ô. F., \& Bouhallab, S. (2014). Heating and glycation of $\beta$-lactoglobulin and $\beta$-casein: Aggregation and in vitro digestion. Food Research International, 55, 70-76. https://doi.org/10.1016/j. foodres.2013.10.030.

Poncheewin, W., Hermes, G. D. A., van Dam, J. C. J., Koehorst, J. J., Smidt, H., \& Schaap, P. J. (2020). NG-Tax 2.0: A Semantic Framework for High-Throughput Amplicon Analysis. Frontiers in Genetics, 10. https://doi.org/10.3389/ fgene.2019.01366.

Qu, W., Nie, C., Zhao, J., Ou, X., Zhang, Y., Yang, S., et al. (2018). MicrobiomeMetabolomics Analysis of the Impacts of Long-Term Dietary Advanced-GlycationEnd-Product Consumption on C57BL/6 Mouse Fecal Microbiota and Metabolites. Journal of Agricultural and Food Chemistry, 66(33), 8864-8875. https://doi.org/ 10.1021/acs.jafc.8b01466.

Qu, W., Yuan, X., Zhao, J., Zhang, Y., Hu, J., Wang, J., et al. (2017). Dietary advanced glycation end products modify gut microbial composition and partially increase colon permeability in rats. Molecular Nutrition and Food Research, 61(10), 1700118. https://doi.org/10.1002/mnfr.201700118.

Quast, C., Pruesse, E., Yilmaz, P., Gerken, J., Schweer, T., Yarza, P., et al. (2013). The SILVA ribosomal RNA gene database project: Improved data processing and webbased tools. Nucleic Acids Research, 41, D590-D596. https://doi.org/10.1093/nar/ gks1219.

Ravussin, Y., Koren, O., Spor, A., Leduc, C., Gutman, R., Stombaugh, J., et al. (2012). Responses of gut microbiota to diet composition and weight loss in lean and obese mice. Obesity, 20(4), 738-747. https://doi.org/10.1038/oby.2011.111.

Roncero-Ramos, I., Delgado-Andrade, C., Haro, A., Ruiz-Roca, B., Morales, F. J., \& Navarro, M. P. (2013). Effects of dietary bread crust Maillard reaction products on calcium and bone metabolism in rats. Amino Acids, 44(6), 1409-1418. https://doi. org/10.1007/s00726-011-1160-3.

Scheijen, J., Hanssen, N. M. J., van Greevenbroek, M. M., Van der Kallen, C. J., Feskens, E. J. M., Stehouwer, C. D. A., et al. (2018). Dietary intake of advanced glycation endproducts is associated with higher levels of advanced glycation endproducts in plasma and urine: The CODAM study. Clin Nutr, 37(3), 919-925. https://doi.org/10.1016/j.clnu.2017.03.019.

Scheijen, J. L. J. M., Clevers, E., Engelen, L., Dagnelie, P. C., Brouns, F., Stehouwer, C. D. A., et al. (2016). Analysis of advanced glycation endproducts in selected food items by ultra-performance liquid chromatography tandem mass spectrometry: Presentation of a dietary AGE database. Food Chemistry, 190, 1145-1150. https://doi.org/10.1016/j.foodchem.2015.06.049.

Segata, N., Izard, J., Waldron, L., Gevers, D., Miropolsky, L., Garrett, W. S., et al. (2011). Metagenomic biomarker discovery and explanation. Genome Biology, 12(6), R60. https://doi.org/10.1186/gb-2011-12-6-r60.

Seiquer, I., Rubio, L. A., Peinado, M. J., Delgado-Andrade, C., \& Navarro, M. P. (2014). Maillard reaction products modulate gut microbiota composition in adolescents. Molecular Nutrition \& Food Research, 58(7), 1552-1560. https://doi.org/10.1002/ mnfr. 201300847.

Snelson, M., Tan, S. M., Clarke, R. E., De Pasquale, C., Thallas-Bonke, V., Nguyen, T. V., et al. (2021). Processed foods drive intestinal barrier permeability and microvascular diseases. Science Advances, 7(14), 1-15. https://doi.org/10.1126/sciadv.abe4841.

Somoza, V., Wenzel, E., Weiß, C., Clawin-Rädecker, I., Grübel, N., \& Erbersdobler, H. F. (2006). Dose-dependent utilisation of casein-linked lysinoalanine, N(epsilon)fructoselysine and N(epsilon)-carboxymethyllysine in rats. Molecular Nutrition \& Food Research, 50(9), 833-841. https://doi.org/10.1002/mnfr.200600021.

Sowndhar Rajan, B., Manivasagam, S., Dhanusu, S., Chandrasekar, N., Krishna, K., Kalaiarasu, L. P., et al. (2018). Diet with high content of advanced glycation end products induces systemic inflammation and weight gain in experimental mice: Protective role of curcumin and gallic acid. Food and Chemical Toxicology, 114, 237-245. https://doi.org/10.1016/j.fct.2018.02.016.

Tessier, F. J., Niquet-Leridon, C., Jacolot, P., Jouquand, C., Genin, M., Schmidt, A. M., et al. (2016). Quantitative assessment of organ distribution of dietary protein-bound $13 \mathrm{C}$-labeled $\mathrm{N} \varepsilon$-carboxymethyllysine after a chronic oral exposure in mice. Molecular Nutrition \& Food Research, 60(11), 2446-2456. https://doi.org/10.1002/ mnfr.201600140.

Tilg, H., \& Moschen, A. R. (2014). Microbiota and diabetes: An evolving relationship. Gut, 63(9), 1513-1521. https://doi.org/10.1136/gutjnl-2014-306928.

Uribarri, J., Cai, W., Peppa, M., Goodman, S., Ferrucci, L., Striker, G., \& Vlassara, H. (2007). Circulating glycotoxins and dietary advanced glycation endproducts: two links to inflammatory response, oxidative stress, and aging. J Gerontol A Biol Sci Med Sci, 62(4), 427-433. https://www.ncbi.nlm.nih.gov/pubmed/17452738.

Uribarri, J., Woodruff, S., Goodman, S., Cai, W., Chen, X., Pyzik, R., et al. (2010). Advanced glycation end products in foods and a practical guide to their reduction in the diet. Journal of the American Dietetic Association, 110(6), 911-16 e12. https://doi. org/10.1016/j.jada.2010.03.018.

Waqas, K., Chen, J., van der Eerden, B. C. J., Ikram, M. A., Uitterlinden, A. G., Voortman, T., et al. (2020). Dietary Advanced Glycation End-Products (dAGEs) Intake and Bone Health: A Cross-Sectional Analysis in the Rotterdam Study. Nutrients, 12(8), 2377. https://doi.org/10.3390/nu12082377.

Xie, J., Reverdatto, S., Frolov, A., Hoffmann, R., Burz, D. S., \& Shekhtman, A. (2008). Structural basis for pattern recognition by the receptor for advanced glycation end 
products (RAGE). Journal of Biological Chemistry, 283(40), 27255-27269. https:// doi.org/10.1074/jbc.M801622200.

Yang, S., Wang, G., Ma, Z. feei, Qin, L., Zhai, Y., Yu, Z., Xue, M., Zhang, Y., \& Wan, Z. (2020). Dietary Advanced Glycation End Products-Induced Cognitive Impairment in Aged ICR Mice: Protective Role of Quercetin. Molecular Nutrition \& Food Research, 64(3), 1901019. https://doi.org/10.1002/mnfr.201901019.
Yudkin, J. S., Stehouwer, C. D. A., Emeis, J. J., \& Coppack, S. W. (1999). C-reactive protein in healthy subjects: Associations with obesity, insulin resistance, and endothelial dysfunction: A potential role for cytokines originating from adipose tissue? Arteriosclerosis, Thrombosis, and Vascular Biology, 19(4), 972-978. https://doi. org/10.1161/01.ATV.19.4.972. 